\title{
Estudantes da educação de jovens e adultos na Educação Física no Enem
}

\section{Students of Youngsters and Adults Education in Physical Education in Enem}

\author{
José Junior ${ }^{* * * 1}$, Leandro Sousa ${ }^{* * * * * * *}$, Sonia Moral ${ }^{* * * * *}$, Mark Carvalho ${ }^{* *}$, Nicolino Trompieri-Filho**** \\ *Universidade Estadual do Ceará, ** Universidade Federal do Acre, ${ }^{* * * *}$ Instituto Federal de Educação, Ciência e Tecnologia do Ceará, \\ ***** Universidade Federal do Ceará, ${ }^{* * * * *}$ Universidade de Salamanca
}

\begin{abstract}
Resumo
O estudo teve o objetivo de analisar o desempenho dos candidatos da EJA nos itens de Educação Física do Enem edição de 2014. Participaram da amostra 305 estudantes selecionados aleatoriamente. Foi realizada a comparação de desempenho por sexo, tipo de escola e regiões geográficas do país. Para a análise utilizou-se o teste $t$ de Student e Análise de Variância. A média geral de desempenho é baixa e observou-se diferenças significativas no desempenho entre os sexos, visto que os homens obtiveram notas maiores que as mulheres, mas não quanto ao tipo de escola e entre as regiões do Brasil.

Palavras-chave: Avaliação Educacional, Programas Nacionais, Educação Física, Escola pública, Educação de adultos
\end{abstract}

\begin{abstract}
The study had the aim of analyzing the performance of the EJA candidates in the Physical Education items of the Enem 2014 edition. 305 students randomly selected participated in the sample. The performance by sex, type of school and geographic regions of the country was compared. For the analysis, Student's t-test and Variance Analysis were used. The overall mean performance is low and there were significant differences in performance between the sexes, since men scored higher than women, but not in the type of school and in the regions of Brazil. Keywords: Educational Assessment, National Programs, Physical Education, Public School, Adult Education
\end{abstract}

\section{Introdução}

No Brasil, a Educação Básica para o público jovem e adulto, ou seja, aqueles que não tiveram acesso na idade própria, é assegurada gratuitamente pela Lei 9394 de 1996 que estabelece as Diretrizes e Bases da Educação Nacional - LDBEN (Brasil, 2005). A lei também assegura a oferta de exames pelo governo para fins de reconhecimento (certificação) de habilidades adquiridas por meios informais. Como mostra Machado (2009) após essa lei houve um aumento considerável de matrículas entre 1997 e 2006, passando de quase novecentos mil para quase um milhão e meio, principalmente nas instituições de âmbito municipal.

Tendo em vista essa política, desde 2009 o Governo Federal passou a oferecer certificação de nível médio para os candidatos do Exame Nacional do Ensino Médio (Enem) acima de 18 anos de idade. Deste então tinha-se aumentado o número de candidatos oriundos da Educação de Jovens e Adultos (EJA) que se prestam a essa prova, chegando a mais de um milhão em 2016. Além disso, o exame se constituiu nos últimos anos como o principal meio de ingresso nas Instituições de Educação Superior (IES) brasileiras, uma vez que muitas universidades públicas e privadas estão aderindo o exame como forma de seleção dos alunos. Ocorre também de IES de outros países selecionarem candidatos brasileiros para ingressarem em seus cursos, como é o caso de Portugal (Brasil, 2017).

Ainda em 2009, com o Novo Enem, a Educação Física foi inserida na matriz de referência do Enem. Desde então itens dessa disciplina têm sido contemplados na prova. Apesar de poucas, entre duas e quatro questões exigidas em cada ano (Pontes Jr, Sousa, \& Silva, 2015), contribuem para a nota final. Isso demonstra a relevância desses conteúdos, indicando que sua apropriação pelos candidatos contribui para sua formação geral. Além disso, segundo a Lei 9394/96, a Educação Física é conteúdo obrigatório em todos os níveis da Educação Básica (Brasil, 2005)

No entanto, vale ressaltar que esse grupo de estudantes da Educação de Jovens e Adultos possuem características peculiares. Di Pierro (2008) enfatiza essa especificidade, não só no Brasil, mas em toda a América Latina, em que esse público vem de um contexto social precário, com alto índice de pobreza, principalmente os da zona rural, se agravando ainda mais quando se trata de negros e indígenas. Cabe apontar a necessidade de melhor análise da qualidade do ensino oferecido a esse público, dada a grande heterogeneidade dos interessados.

Por conta disso, Gomes e Carnille (2003) identificaram em estudo com grupos focais com alunos do Ensino Médio regular e da EJA que: a) a EJA é um âmbito escolhido pelos que tinham poucas perspectivas futuras; b) para os que dispendiam de mais condições de estudo e vislumbravam um futuro promissor, mesmo fora de faixa de idade, procuravam o ensino regular e; c) que os certificados da EJA são vistos com menos prestígio. Tais dados corroboram com Rummert e Ventura (2007), em que discutem a educação de adultos com forma de manutenção do status quo, em que a formação de caráter compensatória e aligeirada, por meio de novos 
programas, mas com velhas concepções, apenas contribuem para a permanência da situação econômica das pessoas.

Dessa forma, o Enem torna-se acessível aos estudantes da EJA, no entanto, esses podem não dispor de igualdade de condições para concorrer aos cursos de graduação da IES. Diante disto, este estudo tem o objetivo de analisar e comparar o desempenho dos candidatos da EJA brasileiros nos itens de Educação Física do Enem, edição de 2014, mais especificamente em relação ao sexo, tipo de escola e regiões geográficas do país.

\section{Método}

\section{Amostra}

A população do presente estudo é constituída por 184.699 candidatos brasileiros que estavam estudando no $3^{\circ}$ ano do Ensino Médio na modalidade EJA no ano de aplicação da prova (2014). Esse critério foi utilizado tendo em vista que o exame se propõe a avaliar os estudantes ao final da Educação Básica. Para isso, o cálculo amostral foi realizado considerando o tamanho do efeito médio pelo programa GPower e que foram selecionados 305 candidatos que atendessem aos critérios de inclusão por amostragem aleatória simples. A média de idade dos estudantes foi de 28.92 ( $\mathrm{dp}=9,36 \mathrm{com} \mathrm{CV}$ $=32.36 \%$ ).

Tabela 1.

Características da amostra

\begin{tabular}{llcc}
\hline Variáveis & & $\mathrm{n}$ & $\%$ \\
\hline Sexo & Feminino & 167 & 54.8 \\
& Masculino & 138 & 45.2 \\
& & & \\
Tipo de escola do & Pública & 257 & 84.3 \\
Ensino Médio & Privada & 48 & 15.7 \\
& & & \\
Estado Civil & Solteiro(a) & 217 & 71.1 \\
& Casado(a)/Mora com & 88 & 28.9 \\
& companheiro(a) & & \\
& & 82 & 26.9 \\
Região de & Nordeste & 109 & 35.7 \\
Residência & Sudeste & 41 & 13.4 \\
& Norte & 33 & 10.8 \\
& Sul & 40 & 13.1 \\
\hline
\end{tabular}

Foram incluídos os candidatos brasileiros com idade mínima de 18 anos, que responderam ao sexo, ao tipo de escola e aos itens de Educação Física. Foram excluídos os que responderam possuir algum tipo de deficiência ou ter solicitado atendimento especial nos dias de aplicação da prova e os que participaram da segunda aplicação do exame por determinação judicial, o que ocorreu apenas em uma cidade com o caderno de questões de cor cinza.

\section{Instrumentos e Coleta dos Dados}

O exame é constituído de uma redação e 180 questões, sendo divididas em quatro áreas: Ciências Humanas e suas Tecnologias, que contempla as disciplinas de História, Geografia, Filosofia e Sociologia; Ciências da Natureza e suas Tecnologias, com Biologia, Química e Física e; Matemática e suas Tecnologias; Linguagens e suas Tecnologias, com as disciplinas de Língua
Portuguesa, Língua Estrangeira Moderna (inglês ou espanhol), Artes, Tecnologia da Informação e Comunicação e Educação Física. Além disso, o exame de 2014 possui quatro gabaritos diferentes representados por cores diferentes dos cadernos de questões. Cada área possui 45 itens. Os itens de Educação Física são comuns a todos os cadernos de questões de sua área, mudando apenas a ordem de apresentação. Nessa edição foi exigido dois itens para a disciplina, evento incomum visto que em nas demais provas são 3 ou 4 questões, buscando atender pelo menos 1 questão para cada um dos 3 descritores da Competência 3 que é relativo a Linguagem Corporal (Brasil, 2017).

A prova é organizada e aplicada pelo Instituto Nacional de Estudos e Pesquisas Educacionais Anísio Teixeira (INEP), ocorrendo em vários municípios de todos os estados do Brasil em dois dias, sendo no primeiro dia as áreas de Ciências Humanas e suas Tecnologias e Ciências da Natureza e suas Tecnologias, no segundo dia as duas últimas mais a redação. As provas utilizadas e os resultados estão disponíveis em forma de microdados para download no site do INEP.

\section{Análise}

Para análise foi considerado as seguintes variáveis: sexo, estado civil, tipo de escola (pública ou privada) e estado da federação em que reside o candidato e suas respostas aos itens de Educação Física. As respostas foram dicotomizadas em certo e errado e depois transformadas em nota dos itens na escala de 0 a 10 para fins de análise e compreensão. Utilizou-se de estatística descritiva (frequência, média e desvio padrão). Também se dispôs de comparação dos resultados entre os grupos através do teste " $t$ " de Student para amostras independentes e Análise de Variância (ANOVA), apresentado o respectivo poder de efeito $r$. Foram considerados os valores de significância de $\mathrm{p} \leq 0,05$. Para tanto, utilizou-se do software SPSS (versão 20.0).

\section{Resultados}

Na Tabela 2 é apresentada média do desempenho dos estudantes por sexo, tipo de escola e região de residência dos candidatos.

Em termos médios, os candidatos do sexo masculino, de escola privada e da região centro-oeste, sul e sudeste apresentam melhores resultados. Mas a média geral é considera baixa $(5,36)$ e percebe-se alta variabilidade dos resultados em todas as variáveis analisadas, principalmente entre os grupos que tiveram as menores médias. Chama a atenção os resultados da região Norte e Nordeste com diferenças de 1.33 e 1.15 para a médias das notas da região Sul (a maior média). 
Tabela 2.

Desempenho médio dos estudantes

\begin{tabular}{llccc}
\hline Variáveis & & média & $\mathrm{dp}$ & CV\% \\
\hline \multirow{2}{*}{ Sexo } & Feminino & 4.79 & 3.74 & 78.08 \\
& Masculino & 6.05 & 3.70 & 61.16 \\
Tipo de escola & Pública & 5.25 & 3.73 & 71.05 \\
do Ensino & Privada & 5.94 & 3.95 & 66.50 \\
Médio & & & & \\
& Solteiro(a) & 5.05 & 3.73 & 73.86 \\
Estado Civil & Casado(a)/união & 6.14 & 3.77 & 61.40 \\
& estável & & & \\
& & & & \\
& Nordeste & 5.06 & 3.56 & 70.36 \\
Região de & Sudeste & 5.41 & 3.92 & 72.46 \\
Residência & Norte & 4.88 & 4.25 & 87.09 \\
& Sul & 6.21 & 3.07 & 49.44 \\
& Centro-oeste & 5.62 & 3.79 & 67.44 \\
Nota média geral & & & & \\
& & 5.36 & 3.76 & 70.15 \\
\hline
\end{tabular}

A comparação do desempenho indicou diferença entre o sexo $(\mathrm{p} \leq .05, r=.17)$. O teste também indicou diferença no desempenho em relação ao estado civil ( $\mathrm{p} \leq$ $.05, \mathrm{r}=.13$ ). No entanto, diferenças no desempenho não foram encontradas em relação ao tipo de escola $(p=.23)$ e em relação à região de residência dos candidatos $(\mathrm{p}=$ .57). A variabilidade dos desempenhos foram altas dentro de cada subgrupo das variáveis, sugerindo heterogeneidade nos resultados, mesmo sem diferenças estatisticamente significativas.

\section{Discussão}

Em termos percentuais, no geral, houve baixo desempenho dos estudantes da EJA nos itens de Educação Física do Enem. Por isso que, em 2017, o Exame Nacional para Certificação de Competências de Jovens e Adultos (Encceja), volta a ser aplicado, visto que é um exame específico para a certificação desse público, tanto no ensino médio quanto do fundamental, bem como para Pessoas Privadas de Liberdade (Brasil, 2017). Cabe enfatizar que a Educação Física está presente na Matriz de Referência do Encceja.

Novamente em relação ao Enem, levantamos a hipótese de que o baixo desempenho nas questões de Educação Física pode estar relacionada a própria Lei 9394 de 1996 em que, apesar de tornar obrigatória essa disciplina, faculta a sua matrícula aos alunos que tenha jornada de trabalho maior ou igual a seis horas, que tenha mais de trinta anos de idade e que tenha prole. E essas características são próprias dos estudantes da EJA. Uma vez que possuem maioridade, necessitam muitas vezes trabalhar para manter as mínimas condições econômicas da família. Assim, por já terem uma carga de estudo intensa, podem se eximir de frequentar as aulas dessa disciplina e de assumir mais um compromisso. A situação é ainda mais agravada porque a maioria dos alunos frequenta a escola no período noturno.

Apesar da LDBEN ter amparado a facultatividade à Educação Física aos alunos de cursos noturnos até o ano de 2003, quando é revogada, Fernandes, Rodrigues, e Nardon (2013) relatam ainda ser frequente, atualmente, a dispensa desses alunos nas aulas dessa disciplina. Com isso, os autores relatam que esses alunos ficam em situação de desvantagem no Enem em relação aos demais, uma vez que eles não têm acesso aos conteúdos, aumentando ainda mais a desigualdade de oportunidades entre os estudantes do ensino noturno, que em sua maioria são da EJA, e os do ensino regular.

Dessa forma, contribui ainda mais para a diminuição da entrada desse grupo no Ensino Superior no Brasil. Quando entram, os alunos desse grupo sofrem muitas dificuldades, tais como conhecimentos prévios insuficientes, falta de tempo advindo do trabalho (Silva, 2015). E por conta disso, somado às questões socioeconômicas dessa população, que em sua maioria possuem família e moram na zona rural, em que historicamente possui uma educação inadequada, os alunos acabam se evadindo, mesmo em cursos superiores à distância (Fernandes, Ferreira, Nascimento, Shimoda, $\&$ Teixeira, 2010).

Por outro lado, tendemos a refletir que a mudança de estilo de aula de Educação Física foi demasiada dos anos de 1996 para a atualidade, visto a inserção de aulas teóricas e currículos escolares direcionados para reflexões sobre as práticas corporais no cotidiano (Sousa, Sobral, \& Trompieri Filho), tal como no exemplo da questões 97 que trata sobre o MMA, espetáculo esportivo de artes marciais, mas que foi tratado no Enem com níveis de criticidade sobre os aspectos históricos que as lutas possuem bem como o seu potencial econômico atual.

O boxe está perdendo cada vez mais espaço para um fenômeno relativamente recente do esporte, o MMA. E o maior evento de Artes Marciais Mistas do planeta é o Ultimate Fighting Championship, ou simplesmente UFC O ringue, com oito cantos, foi desenhado para deixar os lutadores com mais espaço para as lutas. Os atletas podem usar as mãos e aplicar golpes de jiujitsu Muitos podem falar que a modalidade é un de ju-jitsu. Muitos tudo, mas isso já ficou no passado: agora, a modalidade tem regras e acompanhamento médico obrigatório para que o esporte apague o estigma negativo. CORREIA, D. UFC: saiba como o MMA nocauteou o bove em oit golpes

O processo de modificação das regras do MMA retrata a tendência de redimensionamento de algumas práticas corporais, visando enquadrá-las em um determinado formato. Qual o sentido atribuído a essas transformações incorporadas historicamente ao MMA?

(4) A modificação das regras busca associar valores lúdicos ao MMA, possibilitando a participação de diferentes populacõos como atividade de lazer.

B As transformações do MMA aumentam o grau de violência das lutas, favorecendo a busca de emoções mais fortes tanto aos competidores como ao público.

C As mudanças de regras do MMA atendem à necessidade de tornar a modalidade menos violenta, visando sua introdução nas academias de ginástica na dimensão da saúde.

D As modificaçőes incorporadas ao MMA têm por finalidade aprimorar as técnicas das diferentes artes marciais, favorecendo o desenvolvimento da modalidade enquanto defesa pessoal.

(E) As transformações do MMA visam delimitar a violência das lutas, preservando a integridade dos atletas e enquadrando a modalidade no formato do esporte de espetáculo

Figura 1. Questão 97 do caderno Azul do Enem de 2014 (Brasil, 2014)

Espera-se que a prova do Encceja seja um instrumento que mantenha o nível de criticidade que proposto na Matriz de referência para as questões de Educação Física, tal como no Enem, mas o nível de exigência desse exame 
específico de certificação para jovens e adultos e pessoas privadas de liberdade apresenta características própria para o seu fim, ou seja, verificar o nível conhecimento para conclusão do Ensino Médio e não seleção para o Ensino Superior, tal como o Enem. Esse é um aspecto do Enem que desfavorecia os candidatos que necessitavam, apenas, finalizar a Educação Básica. Sousa (2017) aponta ainda vários problemas de inadequação de consistência interna dos itens de Educação Física na prova do Enem, o que potencializa o questionamentos sobre as características dessa prova tão ampla atender a necessidades específicas dos que necessitam da certificação. Como o Novo Enem é de 2009, vários ajustes são necessários a cada nova aplicação. Exemplo disso também são as limitações apresentadas no PISA (Fernandez-Cano, 2016), Programa Internacional de Avaliação de Estudantes de 15 anos aplicados em vários países desde 2000 e que ainda necessita de aprimoramentos devido (um dos fatores) as diferenças entre os públicos dos diversos países (Klein, 2011). Portanto, sendo o Enem composto por diferentes públicos e de diferentes regiões, pode-se considerar necessário políticas educacionais e avaliações específicas para o público do EJA, no caso o Encceja.

Outra fator é que os resultados indicam que não há diferenças em relação ao tipo de escola e entre as regiões brasileiras. Diferentemente, no ensino regular há diferenças nos resultados em relação ao tipo de escola (Figueirêdo, Nogueira, \& Santana, 2014), sendo as particulares com melhor desempenho, como em relação à região de residência dos alunos (Viggiano \& Mattos, 2013), sendo a região Sudeste e Sul as que mais se destacam.

Figueirêdo, Nogueira, e Santana (2014) identificaram que os alunos de escola particular que têm melhores condições socioeconômicas como condições de renda familiar, qualidade de escola, localização da moradia e escolaridade dos pais, têm melhores resultados comparados com os de escolas públicas considerando os mesmos fatores. Pontes Jr et al (2016) indicou maiores notas nos itens de Educação Física entre os candidatos ao Enem das regiões Centro-Oeste, Sudeste e Sul, e de escolas particulares.

Apesar desse último estudo ter sido realizado com o público de 15 a 25 anos e com as provas de 2009 a 2013, os níveis de desempenho com o presente estudo possuem afirmações que, guardada as proporções e características dos participantes, se refletem, ou seja, a Educação Física no Enem tem resultados melhores resultados entre os alunos de escolas particulares e das regiões Centro-Oeste, Sudeste e Sul.

Em relação a região Sul ter obtido média maior entre os candidatos do EJA, Viggiano e Mattos (2013) constataram que os candidatos (em geral, não do EJA especificamente) da região Sul e Sudeste tiveram melhor média em termos relativos em todas as áreas de exame da prova: Linguagens e suas Tecnologias, Matemática e suas Tecnologias, Ciências Humanas e suas Tecnologias e Ciências da Natureza e suas Tecnologias. No entanto, em redação as médias foram melhor distribuídas entre as regiões. Cabe destacar que o estudo não analisou diferenças estatísticas entre as médias. Também podemos relacionar que essa região apresenta um polo diferenciado de formação de professores de Educação Física, com diversos mestrado e doutorados na área, proporcionando maiores possibilidades de intervenção consistente da área. Exemplo disso que é a utilização de provas de Educação Física em algum dos momentos do processo seletivo para Ensino Superior em três universidade do Paraná Unicentro, UEPG e UEM (Santos, 2014).

\section{Conclusão}

Os resultados indicam que existem diferenças no desempenho dos estudantes da EJA brasileiros nos itens de Educação Física do Enem em relação ao sexo, sendo o grupo masculino melhor. Mas quando se trata do tipo de escola (pública ou privada) não houve diferença, assim como entre as regiões do Brasil, apesar das médias das escolas particulares e da reunião Sul apresentarem notas maiores.

Ressalta-se as limitações desse estudo em relação à quantidade de variáveis utilizadas na análise. Recomenda-se a utilização de maior número de informações tais como condições socioeconômicas, uma vez que esses podem influenciar o rendimento dos alunos. Com isso, análises mais robustas como a regressão múltipla pode auxiliar na análise dos fatores que afetam esses resultados.

\section{Agradecimentos \\ José Junior ${ }^{1}$ : Bolsa PNPD da CAPES.}

\section{Referencias}

Brasil, Senado Federal. (2005). Lei de Diretrizes e Bases da Educação Nacional. Disponível em: https://www2.senado.leg.br/bdsf/bitstream/handle/id/7 0320/65.pdf Acesso em: 12 de julho de 2017.

Brasil, Instituto Nacional de Estudos e Pesquisas Educacionais Anísio Teixeira - Inep. (2014). Exame Nacional do Ensino Médio, caderno de prova Azul. Disponível em: http://download.inep.gov.br/educacao_basica/enem/pr ovas/2014/CAD_ENEM_2014_DIA_2_07_AZUL.pd f Acessado em: 12 de julho de 2017

Brasil, Instituto Nacional de Estudos e Pesquisas Educacionais Anísio Teixeira - Inep. (2017). Novo ENEM. Disponível em: <http://portal.mec.gov.br/busca-geral/179-estudantes108009469/vestibulares-1723538374/13318-novoenem>. Acesso em: 12 de julho de 2017.

Brasil, Instituto Nacional de Estudos e Pesquisas Educacionais Anísio Teixeira - Inep. (2017). ENCCEJA. Disponível em: http://portal.inep.gov.br/encceja Acesso em: 12 de julho de 2017.

Di Pierro, M. C. (2008). Educação de jovens e adultos na América Latina e caribe: trajetória recente. Cadernos de Pesquisa, 38(134):367-391. http://dx.doi.org/10.1590/S010015742008000200006.

Fernandes, A., Rodrigues, H. A., \& Nardon, T. A. (2013). A inserção dos conteúdos de educação física no 
ENEM: entre a valorização do componente curricular e as contradições da democracia. Motrivivência, 40:1324.http://dx.doi.org/10.5007/21758042.2013v25n40p13

Fernandes, J., Ferreira, A. S., Nascimento, D. C. O., Shimoda, E., \& Teixeira, G. F. (2010). Identificação de fatores que influenciam na evasão em um curso superior de ensino à distância. Perspectiva online, $4(16)$.

http://www.seer.perspectivasonline.com.br/index.php/ revista_antiga/article/view/464

Fernandez-Cano, A. (2016). A Methodological Critique of the PISA Evaluations. RELIEVE, 22(1):1-16. Doi:http://dx.doi.org/10.7203/relieve.22.1.8806.

Figueirêdo, E., Nogueira, L., \& Santana, F. L. (2014). Igualdade de oportunidades: analisando o papel das circunstâncias no desempenho do ENEM. Revista Brasileira de Economia, 68(3):373-392. http://dx.doi.org/10.1590/S0034-71402014000300005

Gomes, C. A., \& Carnielli, B. L. (2003). Expansão do Ensino Médio: temores sobre a educação de jovens e adultos. Cadernos de Pesquisa, 119, pp.47-69.. http://dx.doi.org/10.1590/S010015742003000200003.

Klein, R. (2011) A reanalysis of PISA results: comparability problems. Ensaio: Avaliação e Políticas Públicas em Educação. 19(73) Rio de Janeiro Oct./Dec. $2011 \quad$ http://dx.doi.org/10.1590/S010440362011000500002

Machado, M. M. (2009). A educação de jovens e adultos no Brasil pós-Lei no 9.394/96: a possibilidade de constituir-se como política pública. Em Aberto, 22(82):17-39. http://dx.doi.org/10.24109/21766673.emaberto.22i82.\%25p

Pontes Jr, J. A. F., Silva, A. G., Tavares, E. D., Sousa, L. A., Bastos, F. A. C., Cruz, F. N. I., Almeida, L. S. (2016). Aspectos psicométricos dos itens de Educação Física relacionados aos conhecimentos de Esporte e Saúde no Exame Nacional do Ensino Médio (ENEM). Motricidade, 12(s1):12-21. Doi: http://dx.doi.org/10.6063/motricidade.8482.

Pontes Jr, J. A. F., Sousa, L. S., \& Silva, A. G. (2015). Itens de educação física no Exame Nacional do Ensino Médio (Enem) via taxonomia de Bloom. Em Anais do VI Congresso Internacional em Avaliação Internacional, pp. 1626-1643, Fortaleza-CE, Brasil. Disponível em:

http://www.nave.ufc.br/vi_ciae/views/documentos/VI \%20CONGRESSO \%20INTERNACIONAL\%20EM $\% 20$ AVALIA\%C3\%87\%C3\%830\%20EDUCACION AL_29\%20OUTUBRO\%202015.pdf>. Acesso em: 14 de julho de 2017.

Rummert, S. M., \& Ventura, J. P. (2007). Políticas públicas para educação de jovens e adultos no Brasil: a permanente (re) construção da subalternidade considerações sobre os Programas Brasil Alfabetizado e Fazendo Escola. Educar em Revista, 29:29-45. http://dx.doi.org/10.1590/S010440602007000100004.

Santos, K. (2014). Os saberes da educação física em pauta: conteúdos de ensino da educação básica $e$ conhecimentos constituintes dos processos de seleção para ingresso no ensino superior. Dissertação (Mestrado). Universidade Estadual do Centro-Oeste, (UNICENTRO), Guarapuava, PR, Brasil. Disponível em:

http://tede.unicentro.br:8080/jspui/bitstream/tede/355/ 1/PR\%20KATIANE\%20DOS\%20SANTOS.pdf>.

Acesso em: 13 de julho de 2017.

Silva, N. (2015). Processo de afiliação de egressos da EJA no Ensino Superior: desafios e propostas à docência universitária. Revista Brasileira de Educação de Jovens $e$ Adultos, 3(5). https://www.revistas.uneb.br/index.php/educajovense adultos/article/view/1389

Sousa, L. A. (2017). Análise psicométrica dos itens de educação física do exame nacional do ensino médio (ENEM) via teoria clássica dos testes. Dissertação (Mestrado). Universidade Federal do Ceará, Programa de Pós-graduação em Educação Brasileira, Fortaleza, CE, Brasil. Disponível em: < http://www.repositorio.ufc.br/bitstream/riufc/22251/1/ 2017_dis_lasousa.pdf $>$. Acesso em: 13 de julho de 2017.

Sousa, L. A., Sobral, A. E. B., \& Trompieri Filho, N. (2015). Educação física em avaliações em larga escala: o caso do Exame Nacional do Ensino Médio - Enem. Revista Educação e Linguagem, 2(1):60-71. Disponível em: < http://www.fvj.br/revista/wpcontent/uploads/2015/10/5_Educ_2015_1.pdf >.

Acesso em: 13 de julho de 2017.

Viggiano, E., \& Mattos, C. (2013). O desempenho de estudantes no Enem 2010 em diferentes regiões brasileiras. Revista Brasileira de Estudos Pedagógicos (online), 94(237):417-438 http://dx.doi.org/10.1590/S2176-66812013000200005 